\title{
Correction to: Female sexual dysfunction in Egyptian women with anxiety: prevalence and patterns
}

\author{
A. E. Arafa $^{1}$ - S. A. Senosy ${ }^{1}$ (D) \\ Published online: 18 July 2018 \\ (C) Springer-Verlag GmbH Germany, part of Springer Nature 2018
}

\section{Correction to: J Public Health: From Theory to Practice https://doi.org/10.1007/s10389-018-0896-6}

In table 4: column 3, row $1-n=78(\%)$ should be $35.0 \pm 7.4$.

The correct table is shown below.

Table 4 Socio-demographic, obstetric and clinical characteristics associated with female sexual dysfunction in the participating women with anxiety

\begin{tabular}{|c|c|c|c|c|}
\hline \multicolumn{2}{|l|}{ Characteristic } & $\begin{array}{l}\text { No dysfunction } \\
n=41(\%)\end{array}$ & $\begin{array}{l}\text { Dysfunction } \\
n=78(\%)\end{array}$ & $\mathrm{p}$ value \\
\hline \multicolumn{2}{|c|}{ Age (mean $\pm \mathrm{sd})$ in years } & $30.1 \pm 5.6$ & $35.0 \pm 7.4$ & $<0.001 *$ \\
\hline \multirow[t]{2}{*}{ Parity } & $\leq 2$ & $20(48.8)$ & $32(41.0)$ & \multirow[t]{2}{*}{0.269} \\
\hline & $>2$ & $21(51.2)$ & $46(59.0)$ & \\
\hline \multirow[t]{2}{*}{ Education } & Literate & $34(82.9)$ & $57(73.1)$ & \multirow[t]{2}{*}{0.165} \\
\hline & Illiterate & $7(17.1)$ & $21(26.9)$ & \\
\hline \multirow[t]{2}{*}{ Menstrual cycle } & Regular & $35(85.4)$ & $43(55.1)$ & \multirow[t]{2}{*}{$0.001 *$} \\
\hline & Irregular & $6(14.6)$ & $35(44.9)$ & \\
\hline \multicolumn{2}{|c|}{ Menarche age $($ mean $\pm s d)$ in years } & $12.7 \pm 1.5$ & $12.9 \pm 1.8$ & 0.447 \\
\hline \multicolumn{2}{|c|}{ Marriage duration (mean $\pm \mathrm{sd}$ ) in years } & $8.6 \pm 4.9$ & $12.3 \pm 6.4$ & $0.002 *$ \\
\hline
\end{tabular}

*p value significant

The original article has been corrected.

The online version of the original article can be found at https://doi.org/ 10.1007/s10389-018-0896-6

\footnotetext{
S. A. Senosy

shoshoahmed80@yahoo.com; shaymaa.sayed@med.bsu.edu.eg

1 Department of Public Health and Community Medicine, Faculty of Medicine, Beni-Suef University, Beni-Suef, Mukbel 62511, Egypt
} 